\title{
NUMERICAL STUDY OF CONVECTIVE HEAT TRANSFER IN AN INCLINED POROUS ENCLOSURE SATURATED WITH NANOFLUID
}

\author{
JANJA KRAMER STAJNKO ${ }^{1}$, RENATA JECL ${ }^{1} \&$ JURE RAVNIK $^{2}$ \\ ${ }^{1}$ Faculty of Civil Engineering, Transportation Engineering and Architecture, University of Maribor, Slovenia \\ ${ }^{2}$ Faculty of Mechanical Engineering, University of Maribor, Slovenia
}

\begin{abstract}
The addition of nanoscale particles into the fluid is recently a common technology used in several industrial processes, since it has been proved that by introducing particles into the working fluid, the heat transfer characteristics can be improved crucially. However, the understanding of fundamental characteristics of nanofluid saturated porous media domains is still limited. The paper presents a numerical study of free convection in a porous enclosure saturated with a nanofluid. A single-phase mathematical model has been employed assuming that the suspension of nanoparticles in fluid can be modelled as a new fluid with effective properties. Fluid flow in porous media is modelled with the macroscopic Navier-Stokes equations, where the governing parameters are averaged over the representative elementary volume. The obtained set of partial differential equations is solved with use of the numerical code based on the Boundary Element Method, which was primarily developed for pure fluid flow applications and was already proved to be efficient for solving several problems of fluid mechanics. Numerical results for different values of governing parameters are obtained, focusing on the effect of different volume fractions of added nanoparticles and different inclination angles of the porous enclosure on the overall heat transfer through porous domain.
\end{abstract}

Keywords: porous media, nanofluid, natural convection, Brinkman-Forchheimer formulation, Boundary Element Method.

\section{INTRODUCTION}

Convective heat transfer in porous media domains has been studied extensively because of its wide range of applications in engineering, nature and environment, such as e.g. groundwater flow, insulation systems, and heat exchangers. In published studies, different mathematical models have been used to describe buoyancy driven flow in porous medium. The Darcy's law is most commonly used mathematical model for the governing momentum equation and is valid for the laminar flow regime $(\operatorname{Re}<10)$ where the velocities are low and the viscous forces dominate over inertial forces. Extension of the governing momentum equations has been made by analogy with the Navier-Stokes equations with addition of the Brinkman term in order to consider the viscous diffusion and the Forchheimer term to study the inertia effects on the free convection [1].

The mixture of nano-scale particles (e.g. metals, oxides, carbides) and a base fluid (e.g. water, oil or ethylene glycol) is called a nanofluid and is an innovative technique for improving the efficiency of cooling and heating processes in several industrial applications such as refrigerators, electronics, biomedicine. Comprehensive reviews for the recent improvements in this field can be found in [2] and [3]. Nanofluids can be mathematically modelled using a single-phase or two-phase approach. In the single-phase approach it is assumed, that the nanoparticles act in the same way as water molecules and have the same local velocities. The assumption is valid only for low concentrations of nanoparticles (2.5$5 \%$ ) and for solid particles of size $<1-100 \mathrm{~nm}$ [4]. However, the two-phase model is more suitable choice to describe two-phase mixtures of nanoparticles and base fluid, since it is physically more correct. This model takes into account some mechanisms which describe 
relative motion between the fluid and nanoparticles, such as Brownian diffusion, thermophoresis [5].

Several numerical techniques have been used to solve the heat transfer problem in porous media and to simulate the fluid flow, however, the most commonly used are the Finite Element Method, the Finite Difference Method and the Finite Volume Method. Recently, the Boundary Element Method (BEM) has been used as an alternative to other methods, mainly because it is very efficient in case when solving potential problems of fluid mechanics e.g. inviscid fluid flow, heat conduction. In this case the mathematical transformation of the governing set of nonlinear partial differential equations results in boundary integral equations only. However, when dealing with non-homogenous and nonlinear problems as e.g. diffusion-convection problems, the domain integrals also occur in the integral representation, which demands the extension of the classical BEM in order to additionally deal the problem within the domain. The main issue in this case is the evaluation of the domain matrices, which are fully populated and unsymmetrical and require a lot of storage space. The numerical algorithm used in the present study is separated into the single- and sub-domain BEM. The kinematic part is solved by the single domain BEM, while the sub-domain BEM is used to solve the equations of the diffusion-advection type [6].

In the present study, some numerical results for the problem of convective flow in an inclined porous enclosure saturated with $\mathrm{Cu}$ nanofluid are presented, gained using an algorithm based on the BEM. The fluid flow in porous media is modelled using the Brinkman-Forchheimer momentum equation. A single-phase nanofluid mathematical model is used, since only low concentrations of nanoparticles $(2.5-5 \%)$ are considered. The numerical code was proven to be efficient on several applications of pure fluid flow e.g. [6], [7] as well as porous media flow [8]-[10]. The influence of the inclination angle and nanoparticle volume fraction on the convective heat transfer have been analyzed.

\section{GOVERNING EQUATIONS}

The problem of convective flow in nanofluid-saturated porous media can be mathematically described with macroscopic conservation equations for mass, momentum and energy which are suitable averaged over the representative elementary volume [11]. The conservation of mass for incompressible fluid is given with the continuity equation:

$$
\vec{\nabla} \cdot \vec{v}=0,
$$

where $\vec{v}$ is volume averaged velocity vector.

The Brinkman-Forchheimer momentum equation is used, which reads as:

$$
\frac{1}{\phi} \frac{\partial \vec{v}}{\partial t}+\frac{1}{\phi^{2}}(\vec{v} \cdot \vec{\nabla}) \vec{v}=\frac{1}{\rho_{n f}} \vec{\nabla} p-\beta_{n f}\left(T-T_{0}\right) \vec{g}+\frac{1}{\phi} \frac{\mu_{n f}}{\rho_{n f}} \nabla^{2} \vec{v}-\frac{1}{K} \frac{\mu_{n f}}{\rho_{n f}} \vec{v}-\frac{F \vec{v}|\vec{v}|}{K^{1 / 2}}
$$

where $\phi$ is porosity, $t$ time, $p$ pressure, $T$ temperature, $\vec{g}$ gravitational acceleration, $K$ permeability, $F$ Forchheimer coefficient, $\rho_{n f}$ density of nanofluid, $\beta_{n f}$, nanofluid thermal expansion coefficient and $\mu_{n f}$ dynamic viscosity of nanofluid. There are two viscous and two inertial terms in the momentum equation:

- The Brinkman viscous term (third on the right hand side) considers the viscous forces and enables to satisfy the non-slip boundary conditions on a boundary. It is analogous to the Laplacian term in the Navier-Stokes equations written for pure fluid flow [1].

- The Darcy term (fourth on the right hand side) is a linear term where the relationship between the velocity field and pressure difference is linked through the fluid viscosity 
and permeability $K$ which depends on the geometry of the porous medium and is a second order tensor in general. When assuming an isotropic porous media, the permeability is a scalar.

- The Forchheimer term (last on the right hand side) describes the nonlinear influences at higher velocities. $F$ is the Forchheimer coefficient which is depending on the nature of porous medium. According to Ergun model it is given as [1]:

$$
K=\frac{\phi^{3} d_{p}^{2}}{a(1-\phi)^{2}}, \quad F=\frac{b}{\sqrt{a \phi^{3}}},
$$

where $a$ and $b$ are Ergun's constants with values $a=150$ and $b=1.75$, while $d_{p}$ is the average particle size of the bed.

Finally, the energy equation can be written as:

$$
\sigma \frac{\partial T}{\partial t}+(\vec{v} \cdot \vec{\nabla}) T=\frac{k_{e}}{\left(\rho c_{p}\right)_{n f}} \nabla^{2} T
$$

where $\sigma$ is the specific heat ratio $\sigma=\phi+(1-\phi)\left(\rho c_{p}\right)_{p} /\left(\rho c_{p}\right)_{n f},\left(\rho c_{p}\right)_{p}$ and $\left(\rho c_{p}\right)_{n f}$ are heat capacitances of solid and nanofluid phase, respectively. Furthermore, $k_{e}$ is the effective conductivity of porous medium. It is assumed, that the thermal properties of solid matrix and the nanofluid are identical [12], resulting in $\sigma=1$ and $k_{e}=k_{n f}$.

Governing eqns (1), (2) and (3) can be converted into a nondimensional form with introduction of the following dimensionless variables:

$$
\vec{v} \rightarrow \frac{\vec{v}}{v_{0}}, \quad \vec{r} \rightarrow \frac{\vec{r}}{L}, \quad t \rightarrow \frac{v_{0} t}{L}, \quad \vec{g} \rightarrow \frac{\vec{g}}{g_{0}}, \quad p \rightarrow \frac{p}{p_{0}}, \quad T \rightarrow \frac{\left(T-T_{0}\right)}{\Delta T} .
$$

The parameters in the above expressions are: $v_{0}$ characteristic velocity is given with the expression $v_{0}=k_{f} /\left(\rho c_{p}\right)_{f} L, k_{f}$ is the fluid thermal conductivity, $\left(\rho c_{p}\right)_{f}$ is the heat capacity for the fluid phase and $L$ is the characteristic length. Moreover, $T_{0}$ is characteristic temperature $T_{0}=\left(T_{2}-T_{1}\right) / 2$ and $\Delta T$ is characteristic temperature difference $\Delta T=T_{2}-T_{1}$, $p_{0}$ is the characteristic pressure $p_{0}=1 \mathrm{bar}$, while gravitational acceleration is $g_{0}=$ $9.81 \mathrm{~m} / \mathrm{s}^{2}$.

In addition, the velocity-vorticity formulation of the governing equations is proposed by introduction of the vorticity vector, which is by the definition a curl of the velocity field $\vec{\omega}=$ $\vec{\nabla} \times \vec{v}$. The governing set of equations in nondimensional velocity-vorticity formulation can now be written in terms of kinematics equation, the vorticity transport equation and the energy equation as:

$$
\begin{gathered}
\nabla^{2} \vec{v}+\vec{\nabla} \times \vec{\omega}=0, \\
(\vec{v} \cdot \vec{\nabla}) \vec{\omega}=(\vec{\omega} \cdot \vec{\nabla}) \vec{v}-C_{A} \operatorname{Pr} R a_{T} \phi^{2} \vec{\nabla} \times T \vec{g}+C_{B} \operatorname{Pr} \phi \nabla^{2} \vec{\omega}- \\
C_{B} \frac{P r}{D a} \phi^{2} \vec{\omega}-\frac{F}{D a} \phi^{2}|\vec{v}| \vec{\omega}, \\
(\vec{v} \cdot \vec{\nabla}) T=C_{C} \nabla^{2} T .
\end{gathered}
$$

In the above equations parameters $C_{A}, C_{B}$ and $C_{C}$ are presenting the nanofluid properties and are given with expressions: 


$$
C_{A}=\frac{\mu_{n f}}{\mu_{f}} \frac{\rho_{f}}{\rho_{n f}}, \quad C_{B}=\frac{\beta_{n f}}{\beta_{f}}, \quad C_{C}=\frac{\alpha_{n f}}{\alpha_{f}},
$$

where $\alpha_{n f}$ is thermal diffusivity of nanofluid, $\alpha_{n f}=k_{n f} /\left(\rho c_{p}\right)_{n f}$ and $\alpha_{f}$ thermal diffusivity of pure fluid $\alpha_{f}=k_{f} /\left(\rho c_{p}\right)_{f}$. The nanofluid properties are obtained using the expressions given in Section 2.1. For the simulation of the pure fluid flow, the parameters are $C_{A}=C_{B}=$ $C_{C}=1$. Since only steady flow simulations are shown in the present paper, the vorticity and energy transport equations are considered without time derivatives $(\partial \vec{\omega} / \partial t=\partial T / \partial t=0)$.

The nondimensional parameters appearing in the momentum equation are:

- $\quad$ The fluid Rayleigh number $R a_{T}=g \beta_{T} \Delta T L^{3} \rho_{f}\left(\rho c_{p}\right)_{f} /\left(\mu_{f} k_{f}\right)$;

- $\quad$ The Prandtl number $\operatorname{Pr}=\mu_{f} c_{p} / k_{f}$;

- The Darcy number $D a=K / L^{2}$.

Furthermore, the porous Rayleigh number $R a_{P}$ is defined linking the thermal Rayleigh number and Darcy number:

- $R a_{P}=R a_{T} \cdot D a$.

\subsection{Nanofluid properties}

Nanofluid properties are given in terms of relations between pure fluid and pure solid properties. In all subsequent expressions, the indexes $f$ and $s$ stand for the fluid and solid phase respectively.

Firstly, nanofluid solid volume fraction $\varphi$ is defined as the ratio between the volume of solid particles $V_{s}$ and the whole volume of solid particles and fluid $\left(V_{s}+V_{f}\right)$ :

$$
\varphi=\frac{V_{S}}{V_{s}+V_{f}}
$$

Relationships between nanofluid and pure fluid properties are described with models. A comprehensive review of different models can be found in [3]. In this paper it is assumed, that nanoparticles are spherical and all adopted models are valid for the case of small temperature gradients.

Density $\rho_{n f}$ can be given with an expression:

$$
\rho_{n f}=(1-\varphi) \rho_{f}+\varphi \rho_{s}
$$

The effective dynamic viscosity $\mu_{n f}$ can be given according to [13] as:

$$
\mu_{n f}=\frac{\mu_{f}}{(1-\varphi)^{2.5}},
$$

where the effective viscosity does not depend on the nanoparticle type. The heat capacitance of nanofluid can be given as:

$$
\left(\rho c_{p}\right)_{n f}=(1-\varphi)\left(\rho c_{p}\right)_{f}+\varphi\left(\rho c_{p}\right)_{s}
$$

The nanofluid thermal expansion coefficient can be written in a similar way:

$$
(\rho \beta)_{n f}=(1-\varphi)(\rho \beta)_{f}+\varphi(\rho \beta)_{s},
$$

taking into account the definition of $\rho_{n f}$, it follows: 


$$
\beta_{n f}=\beta_{f}\left[\frac{1}{1+\frac{(1-\varphi) \rho_{f}}{\varphi \rho_{S}}} \frac{\beta_{S}}{\beta_{f}}+\frac{1}{1+\frac{\varphi \rho_{s}}{1-\varphi \rho_{f}}}\right] .
$$

The effective thermal conductivity $k_{n f}$ is given with the Wasp model [14] as:

$$
k_{n f}=k_{f} \frac{k_{s}+2 k_{f}-2 \varphi\left(k_{f}-k_{s}\right)}{k_{s}+2 k_{f}+\varphi\left(k_{f}-k_{s}\right)} .
$$

Further assumptions for the used model are: the nanoparticles are in thermal equilibrium with the base fluid and the non-slip boundary condition is considered. The fluid flow is assumed to be laminar, steady, Newtonian and incompressible. The dependency between density and temperature can be described with the Boussinesq approximation as:

$$
\rho_{n f}=\rho_{0}\left(1-\beta_{n f}\left(T-T_{0}\right)\right)
$$

where index 0 refers to a reference state.

\section{BOUNDARY ELEMENT METHOD}

The BEM based algorithm is used to solve the governing set of nonlinear partial differential eqns (6), (7) and (8). In order to determine correct values of boundary vorticity, the algorithm is separated into a single-domain and sub-domain parts, where the kinematics equation is solved with the single-domain BEM and gives the boundary vorticity values. The sub-domain BEM solves the vorticity and energy transport equations for unknown domain vorticity and temperature values. The algorithm was primarily developed for pure fluid flow simulations [6], [7], and was later adopted for nanofluids [15] as well as for porous media flow simulations [8]. The computational scheme results in a fully populated system of equations, which limits the maximum grid size due to memory constraints. This drawback can be mitigated by use of the fast BEM, where sparse approximation of full matrices are used [16]. The main advantage of using the single-domain BEM for the boundary vorticity values is that the algorithm conserves mass in complex geometries, which is not the case when using velocity derivatives to calculate boundary vorticity values.

The main steps of the numerical algorithm can be summarized as follows. At the beginning, the velocity and temperature boundary conditions are required, and have to be given in terms of Dirichlet and Neumann type. In addition, the temperature and temperature flux on the solid walls and the no-slip boundary conditions are prescribed. The vorticity boundary conditions are unknown at the beginning and are calculated using the singledomain BEM on the kinematics equation. The known boundary conditions are used to solve the kinematics eqn (6) for the domain velocity values and energy eqn (8) for the temperature values within the domain. The vorticity values within the domain are obtained using a subdomain BEM on the vorticity transport eqn (7).

\subsection{Integral representation of governing equations}

\subsubsection{Kinematics equation}

The unknown boundary vorticity values are obtained using the single-domain BEM on the kinematics eqn (6) which has to be written in its tangential form: 


$$
\begin{aligned}
c(\vec{\xi}) \vec{n}(\vec{\xi}) \times \vec{v}(\vec{\xi})+\vec{n}(\vec{\xi}) \times \int_{\Gamma} \vec{v} \vec{\nabla} u^{*} \cdot \vec{n} d \Gamma= \\
\vec{n}(\vec{\xi}) \times \int_{\Gamma} \vec{v} \times(\vec{n} \times \vec{\nabla}) u^{*} d \Gamma+\vec{n}(\vec{\xi}) \times \int_{\Omega}\left(\vec{\omega} \times \vec{\nabla} u^{*}\right) d \Omega
\end{aligned}
$$

where $\Omega$ is the computational domain and $\Gamma=\partial \Omega$ is the boundary of the domain, $c(\vec{\xi})$ is geometric factor defined as $c(\vec{\xi})=\theta / 4 \pi, \theta$ is the inner angle with origin in $\vec{\xi}$. If $\vec{\xi}$ lies inside the domain, then $c(\vec{\xi})=1$, if $\vec{\xi}$ lies on a smooth boundary, then $c(\vec{\xi})=1 / 2$. Furthermore, $\vec{n}$ is a vector normal to the boundary and $u^{*}$ is the fundamental solution of the Laplace equation given as:

$$
u^{*}=\frac{1}{4 \pi|\vec{\xi}-\vec{r}|} .
$$

In addition, the sub-domain BEM is used on the kinematics eqn (6) in order to calculate the domain velocity values. The integral equation reads as:

$$
c(\xi) \vec{v}(\xi)+\int_{\Gamma} \vec{v}(\vec{n} \cdot \vec{\nabla}) u^{*} d \Gamma=\int_{\Gamma} \vec{v} \times(\vec{n} \times \vec{\nabla}) u^{*} d \Gamma+\int_{\Omega}\left(\vec{\omega} \times \vec{\nabla} u^{*}\right) d \Omega .
$$

The main advantage of this formulations is, that the obtained integral equation is without the derivatives of the velocity or vorticity fields, which enables that the source point is set to function nodes only. The domain velocity values are calculated based on the known boundary values of the velocity from the initial boundary conditions, while the domain and boundary values of the vorticity are known from the previous iteration.

\subsubsection{Vorticity and energy equations}

In order to derive the integral form of the vorticity and energy equations, the same fundamental solution of the Laplace equation as previously is used. The final integral form of the vorticity transport equation is:

$$
\begin{gathered}
c(\vec{\xi}) \omega_{j}(\vec{\xi})+\int_{\Gamma} \omega_{j} \vec{\nabla} u^{*} \cdot \vec{n} d \Gamma=\int_{\Gamma} u^{*} q_{j} d \Gamma \\
+\frac{1}{\operatorname{Pr}} \frac{1}{C_{B}} \frac{1}{\phi} \int_{\Gamma} \vec{n} \cdot\left\{u^{*}\left(\vec{v} \omega_{j}-\vec{\omega} v_{j}\right)\right\} d \Gamma-\frac{1}{\operatorname{Pr}} \frac{1}{C_{B}} \frac{1}{\phi} \int_{\Omega}\left(\vec{v} \omega_{j}-\vec{\omega} v_{j}\right) \cdot \vec{\nabla} u^{*} d \Omega \\
-R a_{T} \frac{C_{A}}{C_{B}} \phi \int_{\Gamma}\left(u^{*} T \vec{g} \times \vec{n}\right)_{j} d \Gamma-R a_{T} \frac{C_{A}}{C_{B}} \phi \int_{\Gamma}\left(T \vec{\nabla} \times u^{*} \vec{g}\right)_{j} d \Omega \\
+\frac{1}{D a} \phi \int_{\Omega} \omega_{j} u^{*} d \Omega+\frac{F}{\operatorname{Pr} \sqrt{D a}} \frac{1}{C_{B}} \phi|\vec{v}| \int_{\Omega} \omega_{j} u^{*} d \Omega,
\end{gathered}
$$

and finally, the integral form of the energy transport equation reads as:

$$
c(\vec{\xi}) T(\vec{\xi})+\int_{\Gamma} T \vec{\nabla} u^{*} \cdot \vec{n} d \Gamma=\int_{\Gamma} u^{*} q_{T} d \Gamma+\frac{1}{C_{C}}\left[\int_{\Gamma} \vec{n} \cdot\left\{u^{*}(\vec{v} T)\right\} d \Gamma-\int_{\Omega}(\vec{v} T) \cdot \vec{\nabla} u^{*} d \Omega\right] .
$$

In the above equations $q_{j}$ is a component of vorticity flux, while $q_{T}$ is a heat flux. In the sub-domain BEM method a mesh of the entire domain $\Omega$ is made, each mesh element is named a subdomain. All equations are written for each of the subdomains. The field functions and flux across the boundary and within the domain are interpolated using shape functions. The hexahedral subdomains with 27 nodes are used enabling continuous quadratic interpolation of field functions. The field functions on each element are interpolated using continuous quadratic interpolation, while fluxes are interpolated using the discontinuous linear interpolation. With discontinuous interpolation, the definition problems in corners and 
edges are avoided. The final discrete system of equations is over-determined and solved using a least squares solver.

\subsection{Geometry of the problem}

The above developed numerical scheme was tested on an example of two-dimensional rectangular inclined porous enclosure, fully saturated with nanofluid. It is assumed, that porous medium is nondeformable, isotropic, homogenous and that there is no heat transfer between the solid and fluid phase. Two opposite walls are subjected to a temperature differences, while other two walls are adiabatic and impermeable. Since the solver is threedimensional, zero flux boundary conditions are used to model a 2D problem. Geometry of the domain is shown on Fig. 1.

Due to applied temperature gradient, density differences are induced, which results in appearance of thermal buoyancy force. Consequently, a large vortex in the main part of the cavity is produced.

The overall heat transfer through porous media is expected to depend on several nanofluid and porous media properties, such as porosity, permeability, thermal conductivity, heat capacitance, nanofluid solid volume fraction. In order to compare different conditions on the heat transfer characteristics, the wall heat flux is calculated, which is given as a dimensionless Nusselt number:

$$
N u=\frac{k_{n f}}{k_{f}} \int_{\Gamma} \vec{\nabla} T \cdot \vec{n} d \Gamma .
$$

The definition is valid for nanofluids as well as for pure fluids, since there the ratio of thermal conductivities is $k_{n f} / k_{f}=1$.

\section{RESULTS AND DISCUSION}

In the example presented in this paper, the $\mathrm{Cu}$ nanoparticles are added to the water as a base fluid, the properties of both are given in Table 1.

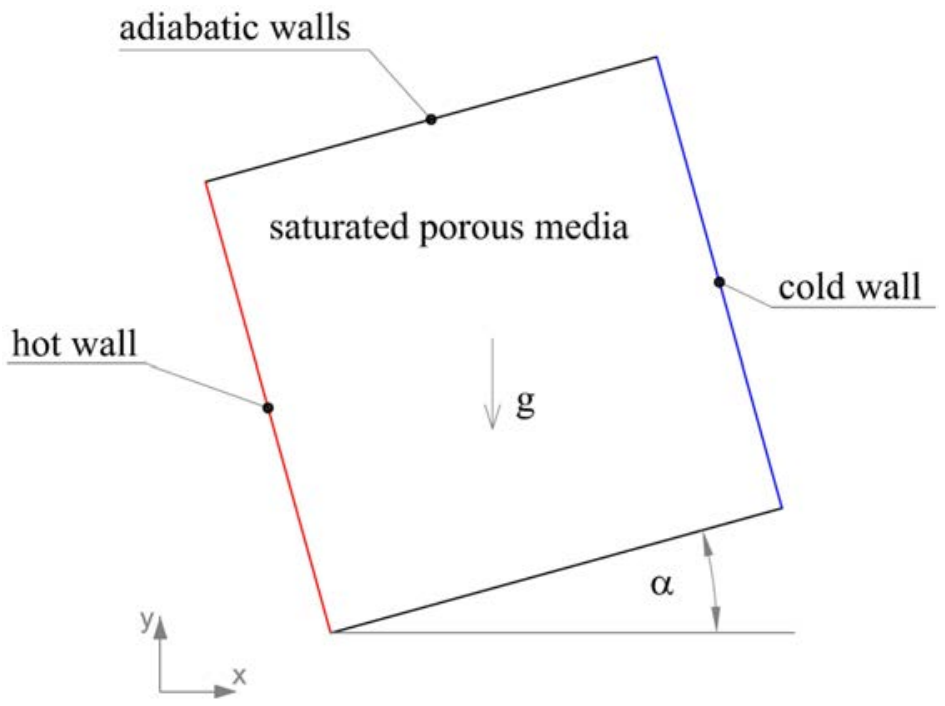

Figure 1: Two-dimensional enclosure with boundary conditions. 
Table 1: Thermophysical properties of water and $\mathrm{Cu}$ nanoparticles.

\begin{tabular}{|c|c|c|c|c|c|}
\hline & $c_{p}[\mathrm{~J} / \mathrm{kg} \mathrm{K}]$ & $\rho\left[\mathrm{kg} / \mathrm{m}^{3}\right]$ & $\mathrm{K}[\mathrm{W} / \mathrm{m} \mathrm{K}]$ & $\beta\left[\times 10^{-5} \mathrm{~K}^{-1}\right]$ & $\alpha\left[\times 10^{-7} \mathrm{~m}^{2} / \mathrm{s}\right]$ \\
\hline Water & 4179 & 997.1 & 0.613 & 21 & 1.47 \\
\hline $\mathrm{Cu}$ & 385 & 8933 & 400 & 1.67 & 1163 \\
\hline
\end{tabular}

The validation of numerical code has been performed on several examples for different geometries and governing parameters. In Table 2 one part of the results is shown in terms of $\mathrm{Nu}$ values for different governing parameters and compared to study [12]. The results are in good agreement with the data from the published study, which confirms accuracy of the obtained numerical algorithm.

Furthermore, heat transfer and fluid flow characteristics have been studied. The influence of the inclination angle and the volume fraction of nanoparticles was investigated. Fig. 2 shows isotherms for $\mathrm{Ra}_{\mathrm{p}}=1000, \operatorname{Pr}=6.2, \mathrm{Da}=10^{-6}, \varphi=0.05$ and inclination angles $0^{\circ}, 15^{\circ}$, $30^{\circ}$ and $60^{\circ}$. With increase of the inclination angle, the overall heat transfer decreases, which results in weaker convection motion. When $\alpha=0^{\circ}$, thin boundary layers are created in the vicinity of the hot and cold walls, almost horizontal isotherms in the core region of the cavity are revealing strong convective motion. In case when $\alpha=60^{\circ}$, convection is supressed and the isotherms become almost linear.

Fig. 3 shows the streamlines for $\phi=0.4, \mathrm{Ra}_{\mathrm{p}}=1000, \operatorname{Pr}=6.2, \mathrm{Da}=10^{-6}, \varphi=0.05$ and different inclination angles. When $\alpha=0^{\circ}$, a single vortex in the clockwise direction can be observed in the flow field, which is a result of applied horizontal temperature differences. Increasing the inclination angle $\left(\alpha>15^{\circ}\right)$, two vortices appear in the middle of the field, and are suppressing the convective motion.

Fig. 4(a) shows the dependence of Nusselt number values on $\mathrm{Ra}_{\mathrm{p}}$ and inclination angles for a Darcy flow regime, when the Darcy number values are low i. e. $\mathrm{Da}<10^{-6}$. Nusselt number values decrease with increase of inclination angles, which is more pronounced at higher values of $\mathrm{Ra}_{\mathrm{p}}$.

Furthermore, Figs 4(b), (c) and (d) show the dependence of $\mathrm{Nu}$ on inclination angle and volume fraction of nanoparticles for $\mathrm{Da}=10^{-6}$ (Darcy flow), $\mathrm{Da}=10^{-4}$, and $\mathrm{Da}=10^{-2}$ (conduction flow regime). Higher values of nanoparticles volume fraction enhance the overall heat transfer through porous media in a conduction regime, when $\mathrm{Da}>10^{-4}$. On the other hand, in the Darcy flow regime $\mathrm{Da}<10^{-6}$, the addition of nanoparticles suppresses the overall heat transfer.

Table 2: Nusselt number values for convective flow in nanofluid saturated porous media for various governing parameters $(\operatorname{Pr}=6.2)$.

\begin{tabular}{|c|c|c|c|c|c|c|c|}
\hline \multirow{2}{*}{$\mathrm{Da}$} & \multirow{2}{*}{$\mathrm{Ra}_{\mathrm{p}}$} & \multicolumn{6}{|c|}{$\phi=0.4$} \\
\cline { 3 - 8 } & & \multicolumn{2}{|c|}{$\varphi=0.0$} & \multicolumn{2}{|c|}{$\varphi=0.025$} & \multicolumn{2}{|c|}{$\varphi=0.05$} \\
\cline { 3 - 8 } & {$[12]$} & Present & {$[12]$} & Present & {$[12]$} & Present \\
\hline $10^{-2}$ & 10 & 1.007 & 1.008 & 1.081 & 1.083 & 1.160 & 1.162 \\
\hline $10^{-2}$ & 1000 & 3.302 & 3.282 & 3.370 & 3.345 & 3.433 & 3.400 \\
\hline $10^{-6}$ & 1000 & 11.867 & 13.238 & 11.847 & 13.131 & 11.778 & 12.991 \\
\hline
\end{tabular}




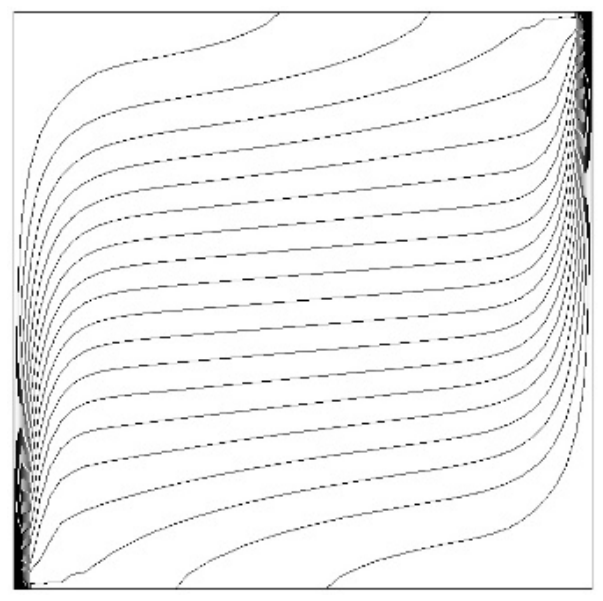

(a)

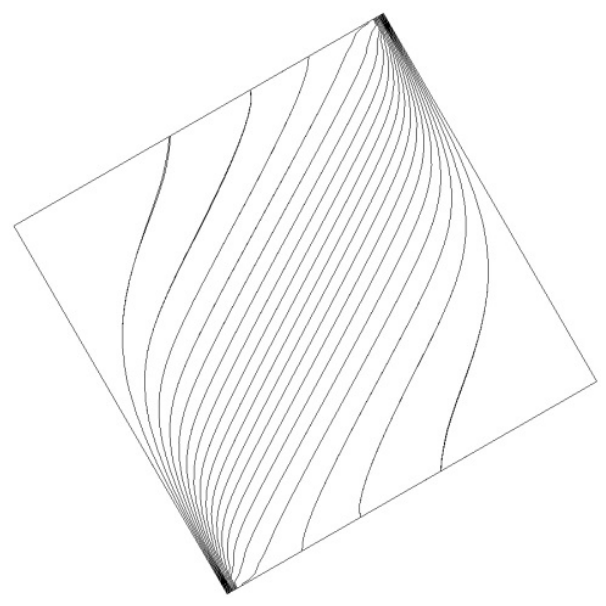

(c)

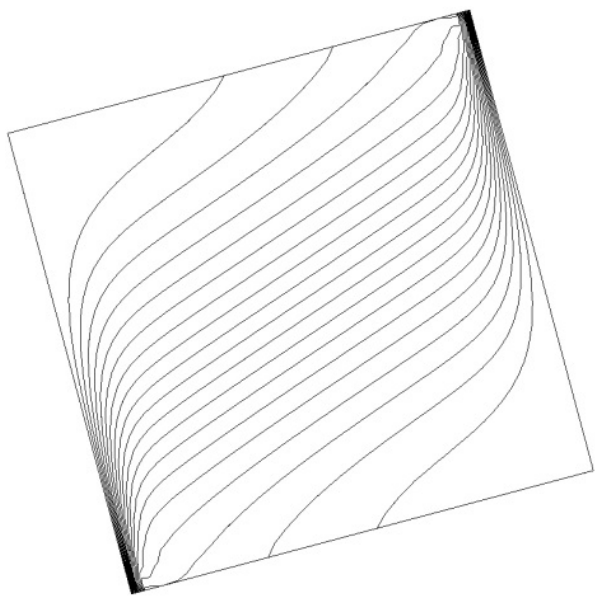

(b)

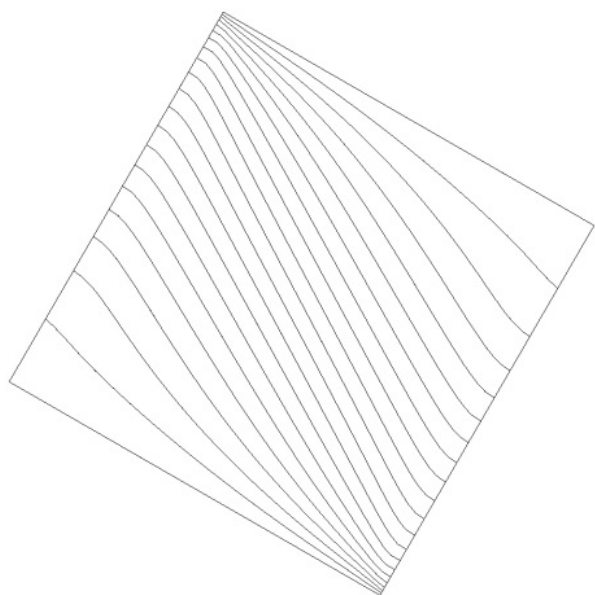

(d)

Figure 2: Isotherms for $\operatorname{Rap}=1000, \operatorname{Pr}=6.2, \mathrm{Da}=10-6, \varphi=0.05$ and (a) $\alpha=0^{\circ}$; (b) $\alpha=15^{\circ}$; (c) $\alpha=30^{\circ}$; (d) $\alpha=60^{\circ}$. 


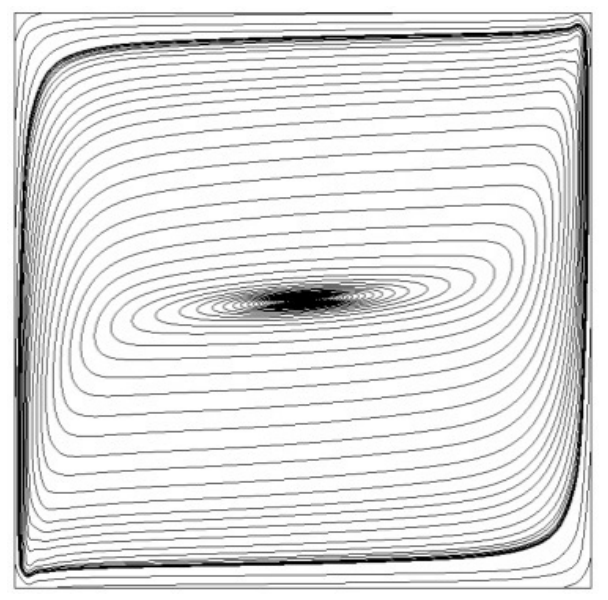

(a)

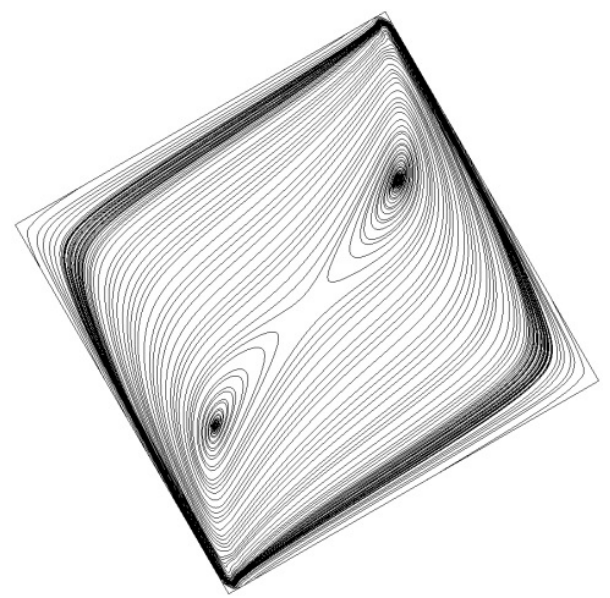

(c)

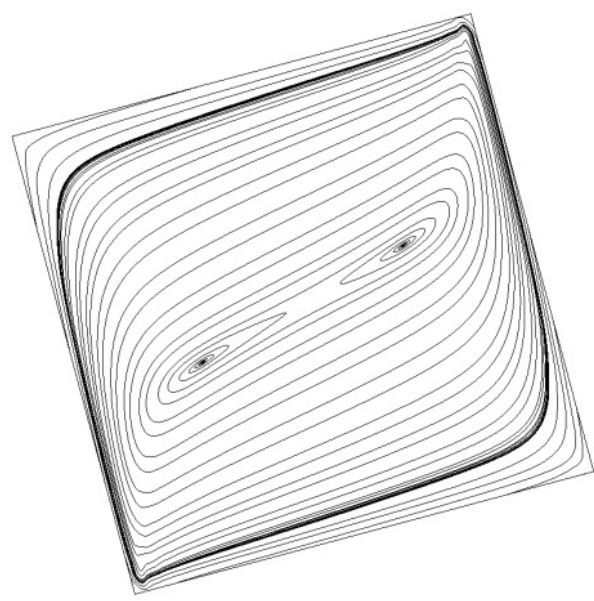

(b)

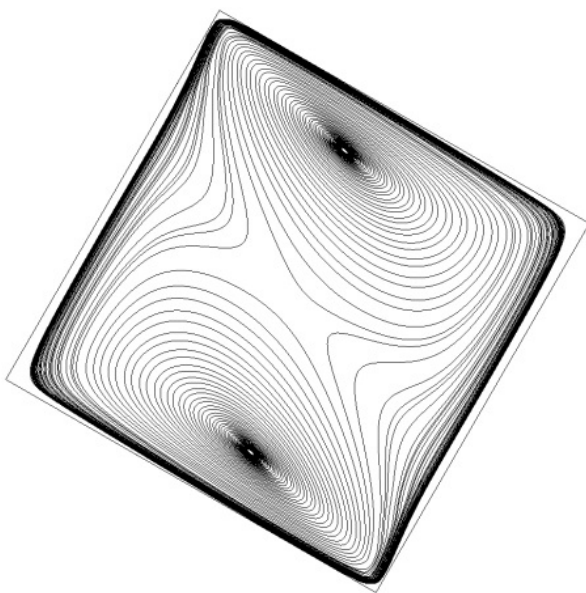

(d)

Figure 3: Streamlines for $\phi=0.4, \operatorname{Rap}=1000, \operatorname{Pr}=6.2, \mathrm{Da}=10-6, \varphi=0.05$ and (a) $\alpha=0^{\circ}$; (b) $\alpha=15^{\circ}$; (c) $\alpha=30^{\circ}$; (d) $\alpha=60^{\circ}$. 


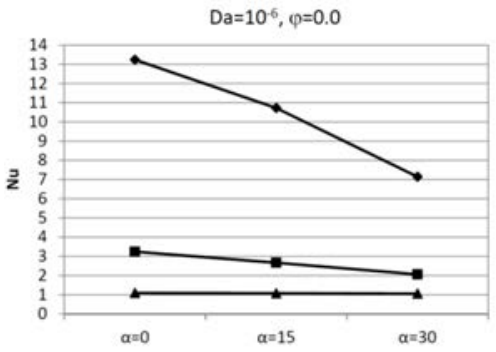

(a)

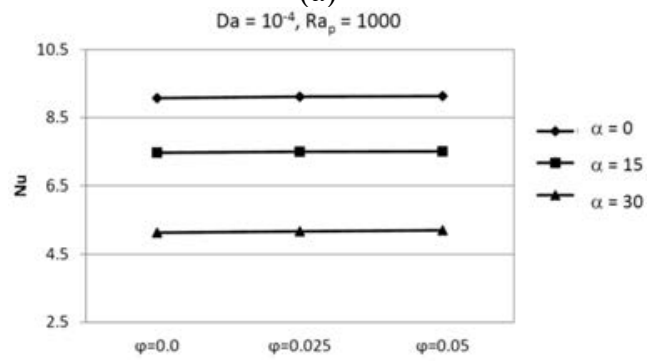

(c)

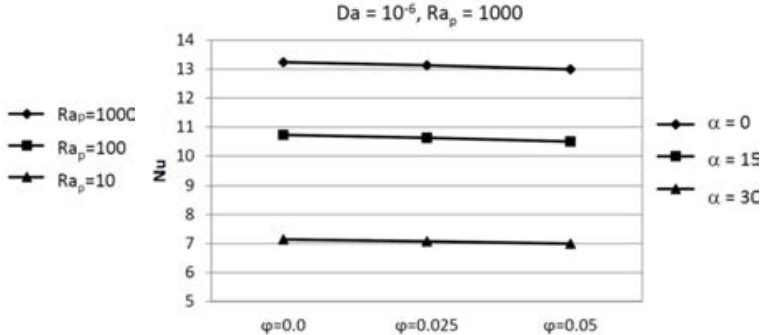

(b)

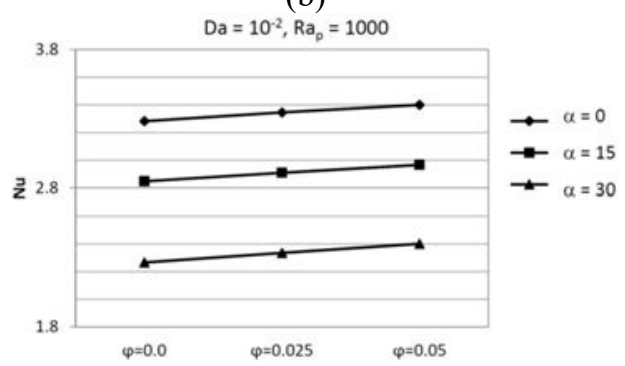

(d)

Figure 4: Nusselt number values depending on solid volume fraction $\varphi$ and inclination angle $\alpha$ for $\phi=0.4, \operatorname{Pr}=6.2$ and (a) $\varphi=0.0, \mathrm{Da}=10-6$; (b) $\mathrm{Rap}=1000, \mathrm{Da}=10-6$; (c) $\operatorname{Rap}=1000, \mathrm{Da}=10-4$; (d) Rap $=1000, \mathrm{Da}=10-2$.

\section{CONCLUSION}

Numerical study based on BEM of convective heat transfer in a differentially heated inclined cavity filled with non-Darcy porous medium fully saturated with nanofluid is presented. Effects of nanofluid solid volume fraction and inclination angle on overall heat transfer and fluid field for different flow regimes were demonstrated. The addition of nanoparticles into a base fluid generally results in higher heat transfer rate. Increasing the solid volume fraction of nanoparticles results in higher values of Nusselt numbers in a conduction regime at high values of Darcy numbers $\left(\mathrm{Da}>10^{-4}\right)$ and low values of Rayleigh numbers $\left(\mathrm{Ra}_{\mathrm{p}}<100\right)$. However, in the Darcy flow regime, where Darcy numbers are low $\left(\mathrm{Da}<10^{-6}\right)$ and Rayleigh numbers high $\left(\mathrm{Ra}_{\mathrm{p}}>1000\right)$, the Nusselt number decrease with increase of the solid volume fraction of nanoparticles. The increase of inclination angle decreases the overall heat transfer through porous cavity, which can be observed for any value of solid volume fraction of nanoparticles.

\section{REFERENCES}

[1] Nield, D.A. \& Bejan, A. (eds), Convection in Porous Media, 4th ed., Springer-Verlag: Berlin and New York, pp. 8-17, 2013.

[2] Ozerinc, S., Kakaç, S. \& Yazicioglu, A.G., Enhanced thermal conductivity of nanofluids: A state-of-the-art review. Microfluid Nanofluid, 8(2), pp. 145-170, 2010.

[3] Haddad, Z., Oztop, H.F., Abu-Nada, E. \& Mataoui, A., A review on natural convective heat transfer of nanofluids. Renew. Sust. Energ. Rev., 16(7), pp. 5363-5378, 2012.

[4] Tiwari, R.K. \& Das, M.K., Heat transfer augmentation in a two-sided lid driven differentially heated square cavity utilizing nanofluids. Int. J. Heat Mass. Tran., 50(910), pp. 2002-2018, 2007. 
[5] Buongiorno, J., Convective transport in nanofluids. J. Heat Transfer, 128(3), pp. 240250, 2006.

[6] Ravnik, J., Škerget, L. \& Žunič, Z., Combined single domain and subdomain BEM for 3D laminar viscous flow. Eng. Anal. Bound. Elem., 33(3), pp. 420-424, 2009.

[7] Ravnik, J., Škerget, L. \& Žunič, Z., Velocity-vorticity formulation for 3D natural convection in an inclined enclosure by BEM. Int. J. Heat Mass. Transfer, 51(17-18), pp. 4517-4527, 2008.

[8] Stajnko Kramer, J., Ravnik, J., Jecl, R. \& Škerget, L., Simulation of 3D flow in porous media by boundary element method. Eng. Anal. Bound. Elem., 35(12), pp. 1256-1264, 2011.

[9] Stajnko Kramer, J., Ravnik, J., Jecl, R. \& Škerget, L., Three-dimensional double diffusive natural convection with opposing buoyancy effects in porous enclosure by boundary element method. Int. J. Computational Methods and Experimental Measurements, 1(2), pp. 103-115, 2013.

[10] Stajnko Kramer, J., Ravnik, J. \& Jecl, R., Natural convection in a square cavity filled with a non-Darcy porous medium saturated with nanofluid by the Boundary Element Method. J. Porous Media, 20(10), pp. 921-939, 2017.

[11] Bear, J., Dynamics of Fluids in Porous Media, Dover Publications: New York, 1972.

[12] Nguyen, M.T., Aly, A.M. \& Lee, S.W., Natural convection in a non-Darcy porous cavity filled with $\mathrm{Cu}$-water nanofluid using the characteristic-based split procedure in finite-element method. Num. Heat. Tr. A-Appl., 67(2), pp. 224- 247, 2015.

[13] Brinkman, H.C., The viscosity of concentrated suspensions and solutions. J. Chem. Phys., 20(4), pp. 571-581, 1952.

[14] Wasp, E.J, Kenny, J.P. \& Gandhi, R.L., Solid-Liquid Flow Slurry Pipeline Transportation, Trans Tech Publications: Clausthal, Germany, 1977.

[15] Ravnik, J. \& Hriberšek, M., Analysis of three-dimensional natural convection of nanofluids by BEM. Eng. Anal. Bound. Elem.,4(12), pp. 1018-1030

[16] Ravnik, J., Škerget, L. \& Žunič, Z., Comparison between wavelet and fast multipole data sparse approximations for Poisson and kinematic boundary-domain integral equations. Comput. Method Appl. M., 198(15), pp. 1473-1485, 2009. 